\title{
Supporting Information: Ligand Length and Surface Curvature Modulate Nanoparticle Surface Heterogeneity and Electrostatics
}

Dongyue Liang, ${ }^{\dagger}$ Udaya Dahal, ${ }^{\ddagger}$ Meng Wu ${ }^{\llbracket}$ Catherine J. Murphy, ${ }^{\llbracket}$ and Qiang $\mathrm{Cui}^{*}, \S$

$\dagger$ Department of Chemistry and Theoretical Chemistry Institute, University of

Wisconsin-Madison, 1101 University Avenue, Madison, WI 53706

$\ddagger$ Department of Chemistry, Boston University, 590 Commonwealth Avenue Boston, MA 02215

9Department of Chemistry, University of Illinois at Urbana-Champaign, Urbana, Illinois 61801, United States

$\S$ Departments of Chemistry, Physics and Biomedical Engineering, Boston University, 590 Commonwealth Avenue Boston, MA 02215

E-mail: qiangcui@bu.edu, Tel:(+1)-617-353-6189 
Table S1: Summary of experimentally measured $T_{2}$ relaxation time of nanoparticles with different core diameters from Meng and co-workers. ${ }^{1}$ a

\begin{tabular}{lcc}
\hline Diameter $(\mathrm{nm})$ & $T_{2}(\mathrm{~ms})$ & $T_{2}^{\star}(\mathrm{ms})$ \\
\hline $1.2 \pm 0.3$ & $20 \pm 2$ & $6.5 \pm 0.4$ \\
$4.8 \pm 1.1$ & $9.8 \pm 1.0$ & $1.0 \pm 0.1$ \\
$6.4 \pm 1.1$ & $8.9 \pm 0.9$ & $1.0 \pm 0.1$ \\
$8.2 \pm 0.9$ & $4.1 \pm 0.5$ & $1.2 \pm 0.1$ \\
$10.8 \pm 0.8$ & $2.4 \pm 0.3$ & $1.3 \pm 0.1$ \\
$13.4 \pm 1.2$ & $1.6 \pm 0.2$ & $1.5 \pm 0.1$ \\
$19.3 \pm 3.0$ & $0.78 \pm 0.10$ & $1.0 \pm 0.1$ \\
$25.0 \pm 4.4$ & $0.60 \pm 0.06$ & $0.6 \pm 0.1$
\end{tabular}

a. $T_{2}$ was determined by the CPMG pulse sequence and $T_{2}^{*}$ was calculated by NMR peak width.

Table S2: Mobilities of different nanoparticles from independent drift velocity simulations with different directions of electric field. ${ }^{a}$

\begin{tabular}{l|cccccc}
\hline Nanoparticle & $-\mathrm{x}$ & $-\mathrm{y}$ & $-\mathrm{z}$ & $+\mathrm{x}$ & $+\mathrm{y}$ & $+\mathrm{z}$ \\
\hline C4Q65 & $9.10 \pm 0.02$ & $9.47 \pm 0.02$ & $9.10 \pm 0.01$ & $9.73 \pm 0.01$ & $9.17 \pm 0.02$ & $8.63 \pm 0.03$ \\
C4Q65 (15nm) & $9.60 \pm 0.02$ & $9.21 \pm 0.01$ & $9.10 \pm 0.04$ & $8.27 \pm 0.01$ & $9.20 \pm 0.01$ & $8.33 \pm 0.02$ \\
C11Q65 (15nm) & $9.30 \pm 0.01$ & $10.40 \pm 0.02$ & $10.63 \pm 0.01$ & $11.14 \pm 0.02$ & $11.84 \pm 0.02$ & $11.61 \pm 0.02$ \\
MTAB65 (15nm) & $9.94 \pm 0.01$ & $10.60 \pm 0.01$ & $12.41 \pm 0.01$ & $11.35 \pm 0.01$ & $11.60 \pm 0.01$ & $11.15 \pm 0.02$ \\
\hline
\end{tabular}

a. The unit of mobility is $10^{-4} \cdot \mathrm{cm}^{2} \cdot \mathrm{V}^{-1} \cdot \mathrm{s}^{-1}$, electric field applied is $0.05 \mathrm{kcal} \cdot \mathrm{mol}^{-1} \cdot \AA^{-1} \cdot \mathrm{e}^{-1}$.

b. Computed using a larger box of $15 \mathrm{~nm}$ size instead of $12 \mathrm{~nm}$. 


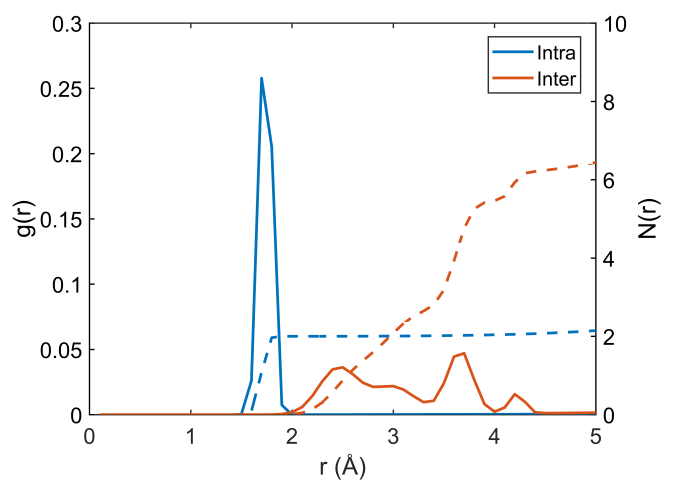

(a)

Figure S1: Radial distribution functions of head group methyl protons from the same/different methyl groups (labeled as intra/inter) in MTAB on a nanoparticle. 


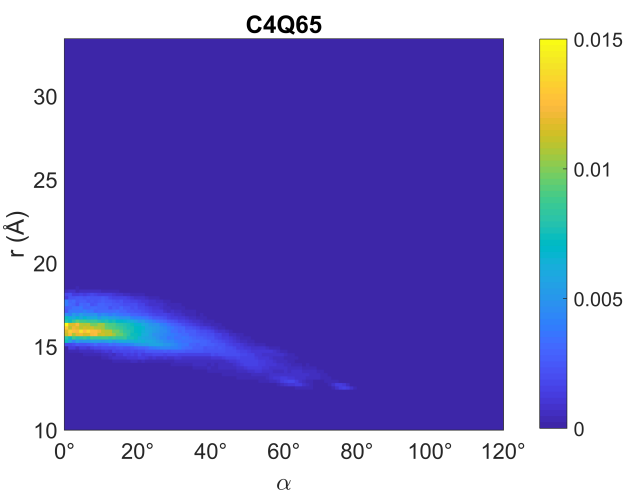

(a)

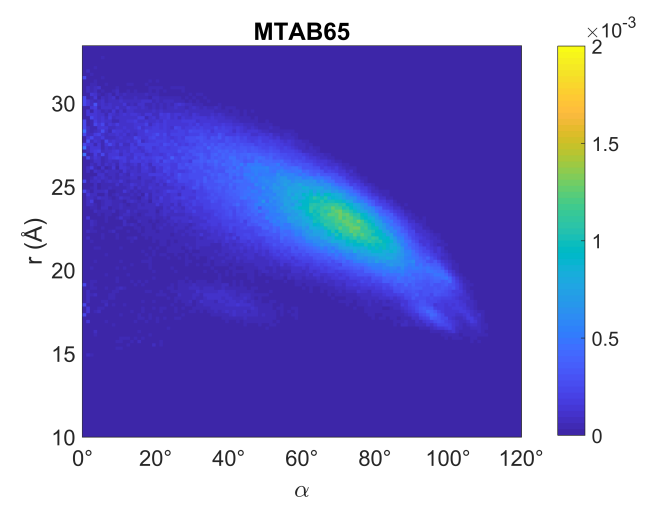

(c)

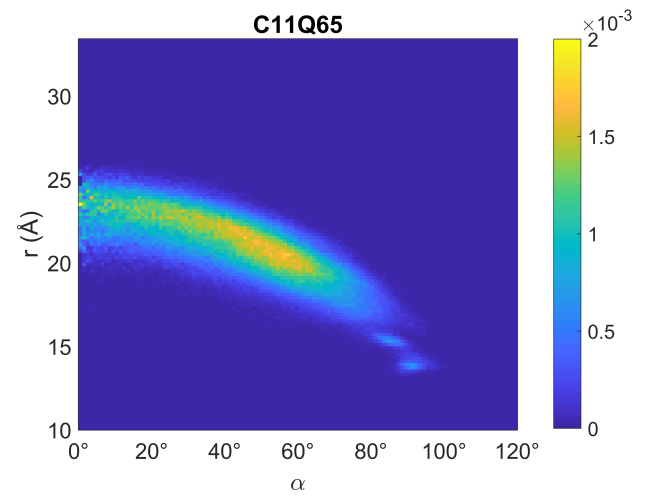

(b)

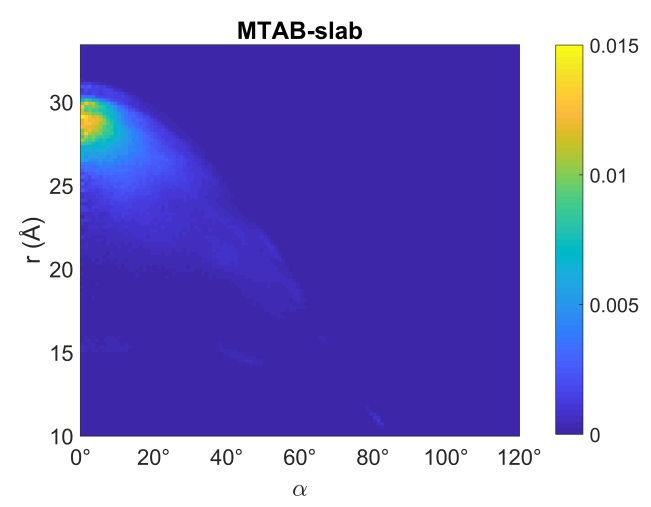

(d)

Figure S2: Two-dimensional joint distributions of radial distance and angular orientation of the ligand head groups nitrogens for nanoparticle and slab cases. The angular distribution is determined by the $\mathrm{S}-\mathrm{N}$ vector relative to the local surface normal and is weighted with the Jacobian factor $\sin \theta$. The distance $r$ is defined relative to the nanoparticle center; for the case of the slab, it is defined along the surface normal and shifted such that the average position of the ligand sulfur aligns with that in the nanoparticle cases.

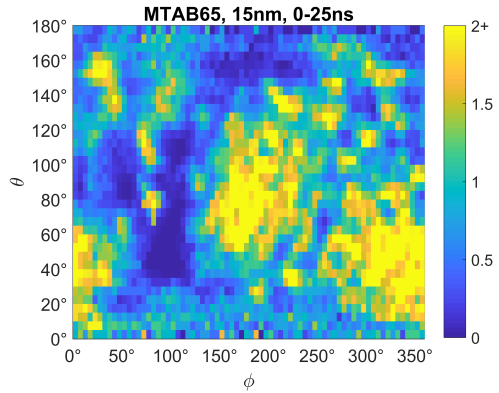

(a)

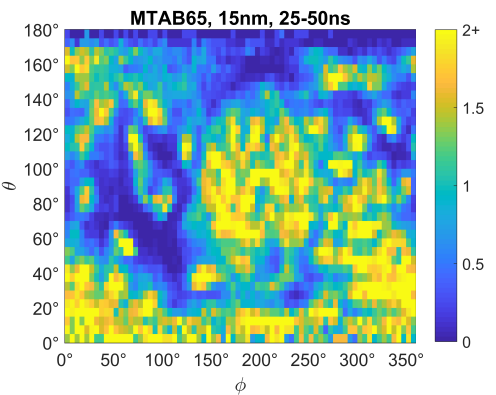

(b)

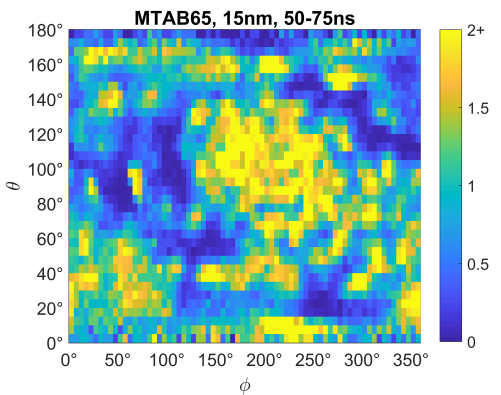

(c)

Figure S3: Same as Fig. 3 in the main text, except that the simulations were conducted with a larger box of $15 \mathrm{~nm}$. 


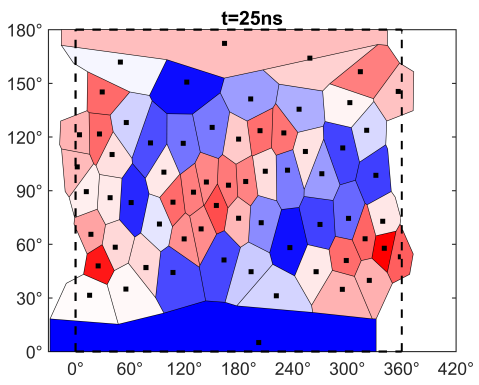

(a)

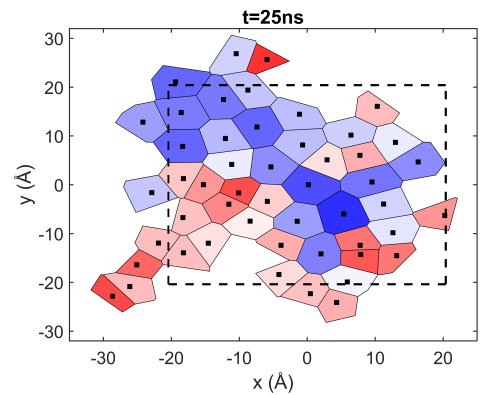

(d)

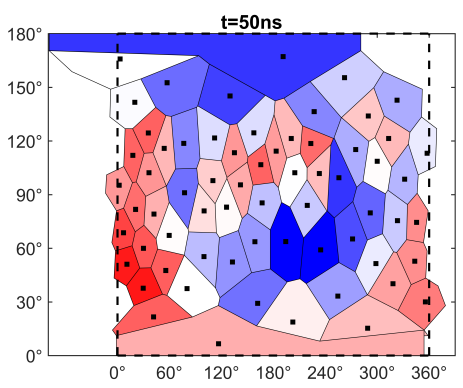

(b)

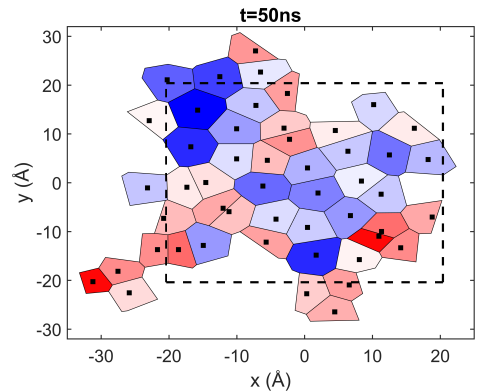

(e)

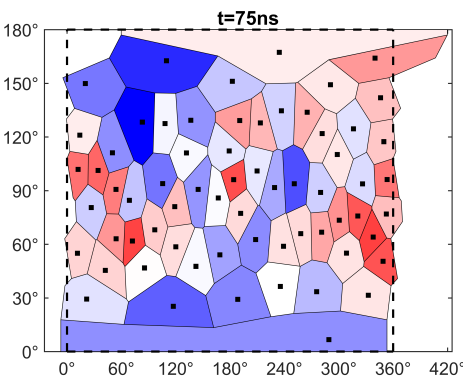

(c)

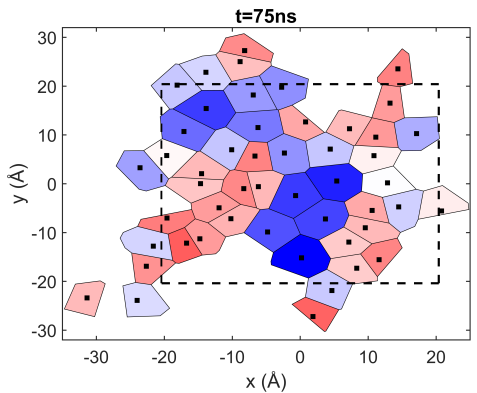

(f)

Figure S4: Same as Fig. 4 in the main text, but for the case of (a-c) C11Q65 and (e-f) MTAB-slab. In the case of MTAB-slab, the dash lines indicate the periodicity inherent in the PBC setup. Voronoi tessellation is done using the software SciPy1.0. ${ }^{2}$

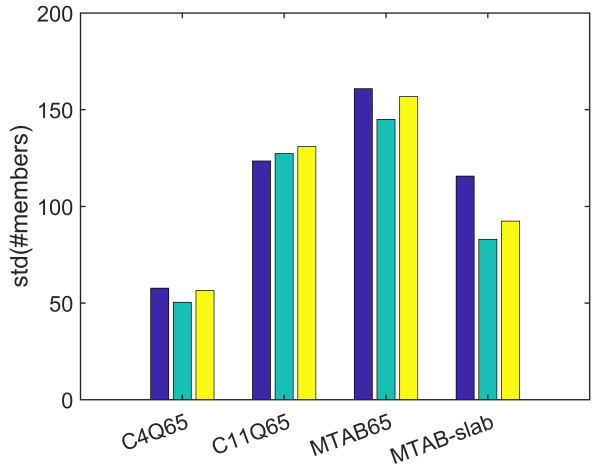

(a)

Figure S5: Standard deviations of cluster sizes from hierarchical cluster analyses ${ }^{2}$ of ligand head group nitrogen positions with 500 frames from segments of 25 ns simulations. For each nanoparticle/slab case, the three bars correspond to analysis from 0-25 ns, 25-50 ns and 50-75 ns, respectively. In each cluster analysis, the max number of clusters allowed is set to be 65/50 for nanoparticle/slab cases, yielding average cluster sizes of 500 members. The results highlight that longer ligands on nanoparticles feature more heterogeneous ligand distributions. 


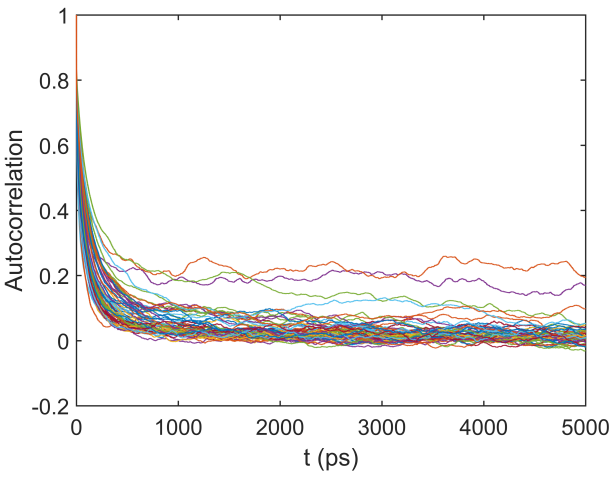

(a)

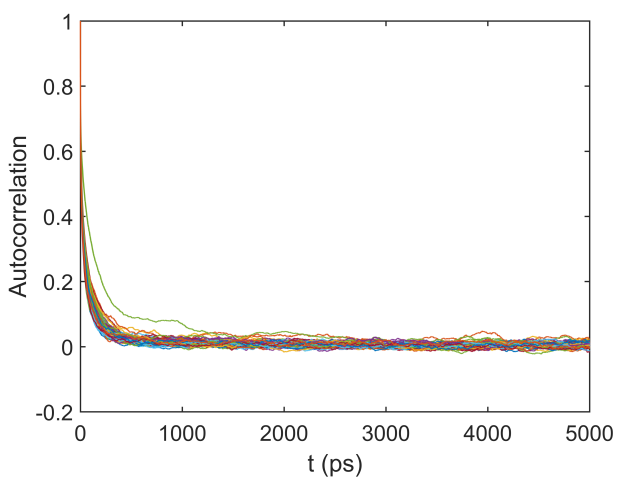

(c)

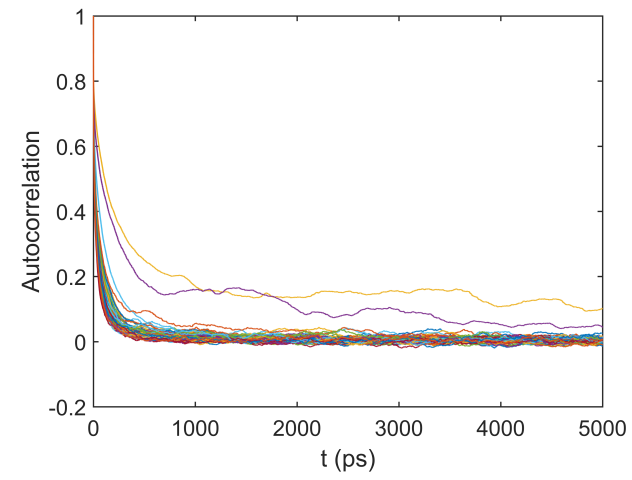

(b)

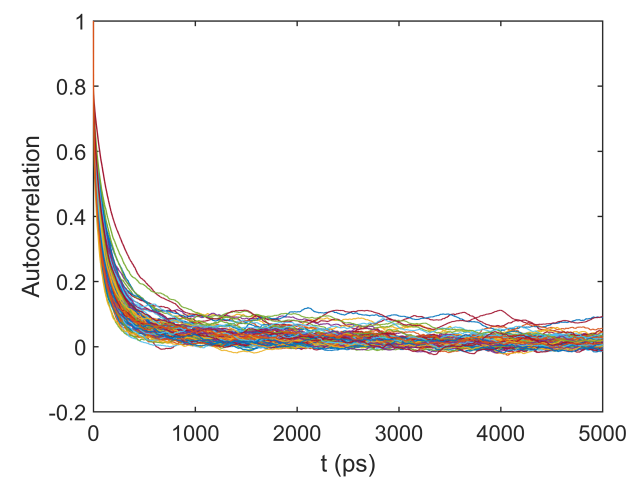

(d)

Figure S6: Same as Fig. 7a in the main text. (a) C4Q65; (b) C11Q65; (c) MTAB65; (d) MTAB-slab. 


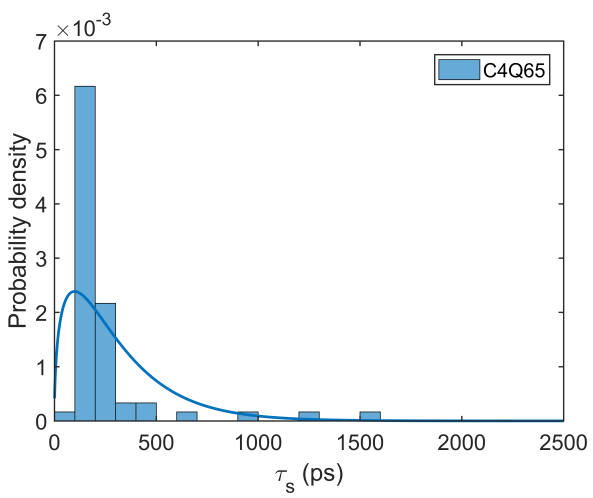

(a)

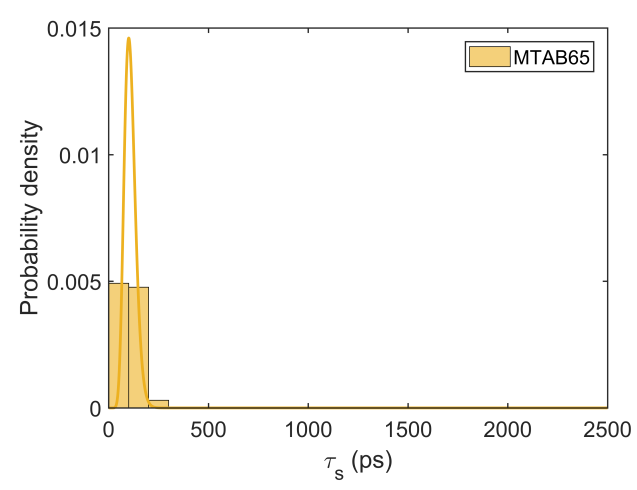

(c)

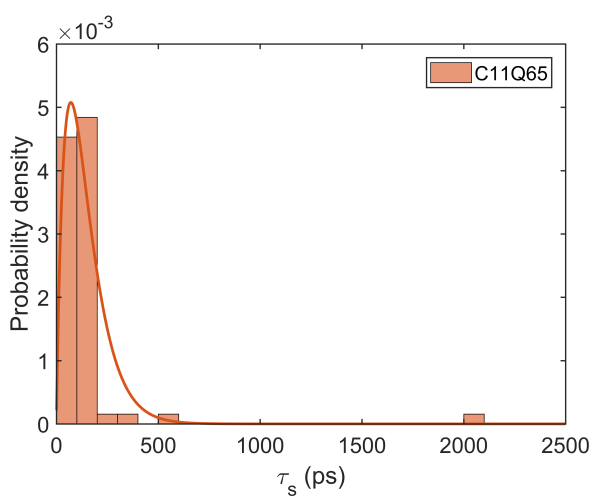

(b)

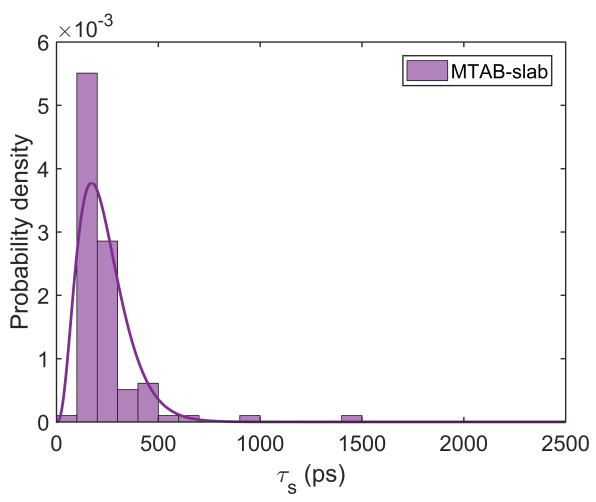

(d)

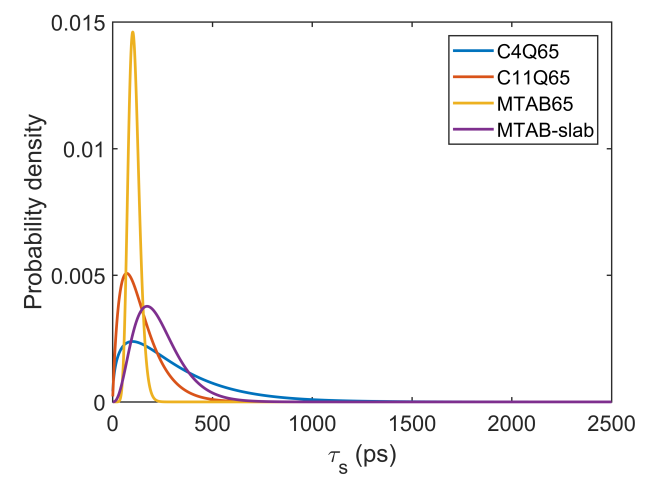

(e)

Figure S7: Comparison of distributions of the slower decay time $\left(\tau_{s}\right)$ from ligand $P_{2}$ autocorrelation functions fitted to $\Gamma$ distributions. (a-d) show the fits of raw $\tau_{s}$ data into $\Gamma$ distributions and (e) compares the fits. For C4Q65, C11Q65, MTAB65 and MTAB-slab cases, the variances associated with each $\Gamma$ distribution are $6.3 \times, 1.1 \times, 0.8 \times$ and $1.4 \times 10^{4}$ $\mathrm{ps}^{2}$, respectively. 


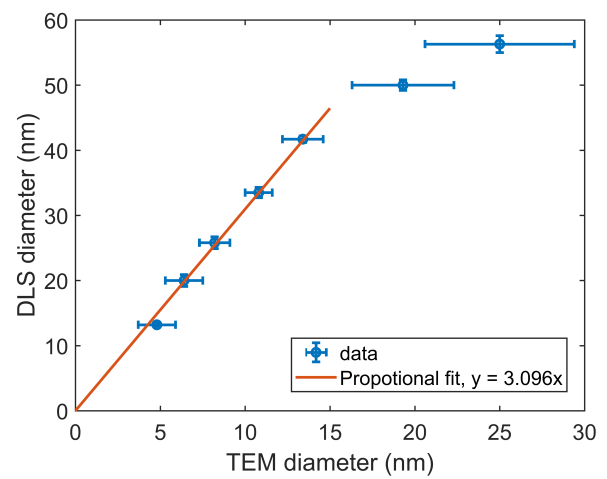

(a)

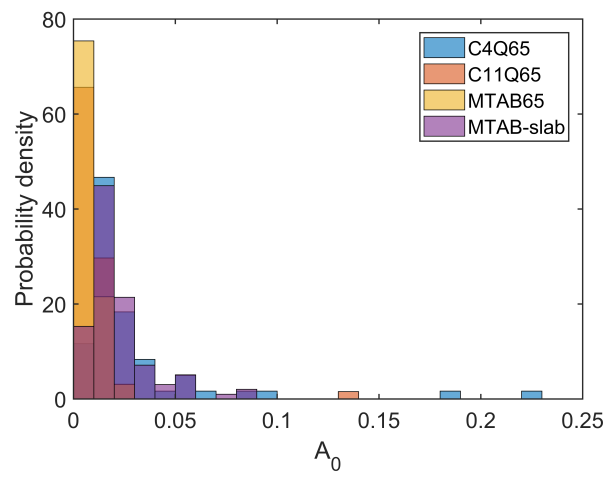

(b)

Figure S8: Quantities relevant to the rotational correlation contribution to the computation of the $T_{2}$ relaxation time. (a) Relationship between measured ${ }^{1}$ hydrodynamic diameter and core diameter of MTAB-functionalized gold nanoparticles by dynamic light scattering (DLS) the transmission electron microscopy (TEM), respectively. The fit for small and moderate sizes of nanoparticles is used to estimate the hydrodynamic diameter of the MTAB65 nanoparticle studied here. (b) The distribution of the order parameter $A_{0}$ in the double exponential fit of the methyl proton $P_{2}$ correlation function (Eq. 3 of the main text); its magnitude determines the contribution of overall rotation to the spectral density (Eq. 5 in the main text). MATB65 has a very narrow distribution with $A_{0}$ value only up to 0.03 .

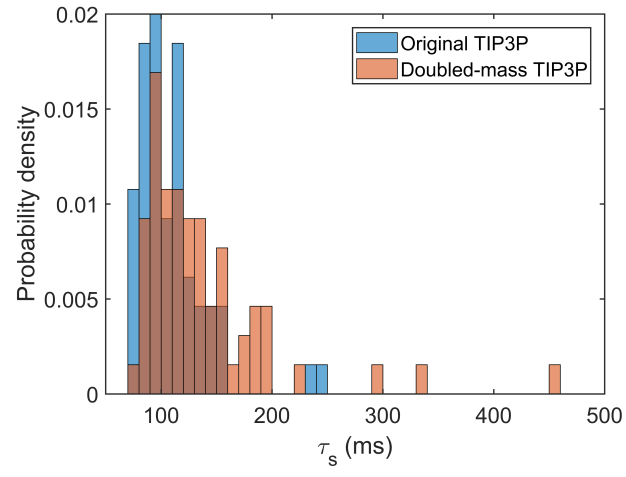

(a)

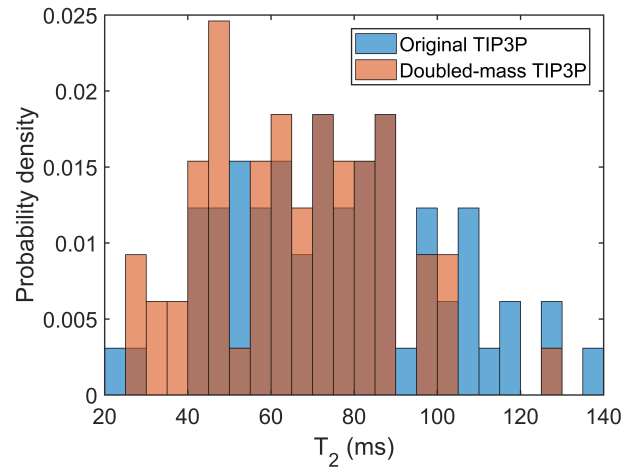

(b)

Figure S9: Comparison of distributions of (a) the slower decay time $\tau_{s}$ of the methyl proton $P_{2}$ correlation function and (b) computed $T_{2}$ relaxation times for the MTAB65 case with the original TIP3P water model vs. the TIP3P water model with doubled atomic masses. 


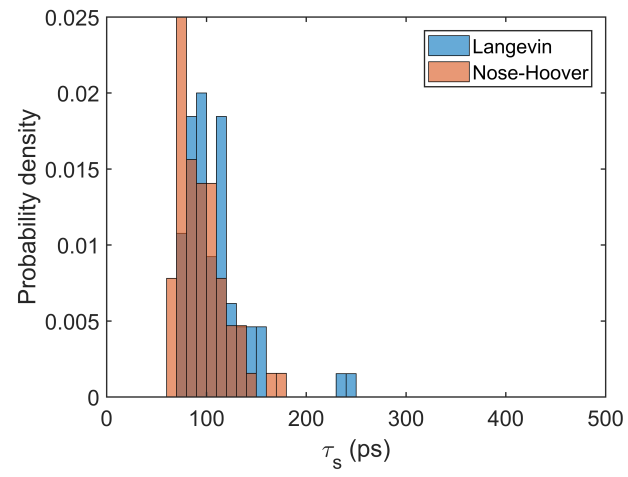

(a)

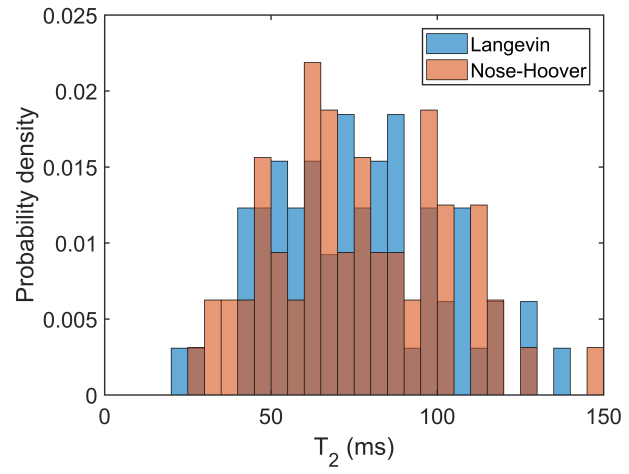

(b)

Figure S10: Comparison of distributions of (a) the slower decay time $\tau_{s}$ of the methyl proton $P_{2}$ correlation function and (b) computed $T_{2}$ relaxation times for the MTAB65 case with different thermostats: Langevin dynamics with $1 \mathrm{ps}^{-1}$ damping coefficient and the NoseHoover thermostat. 


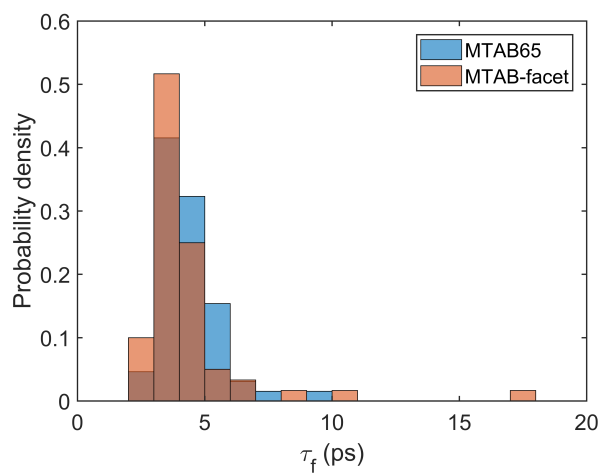

(a)

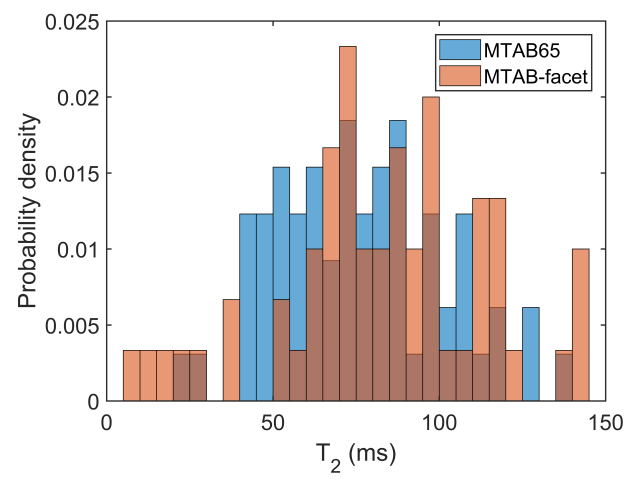

(c)

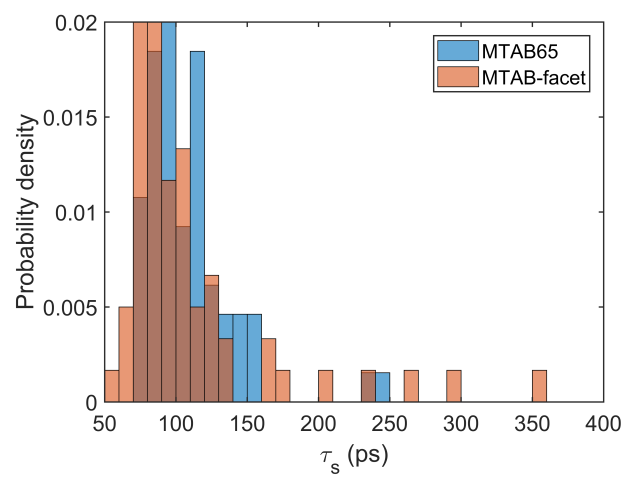

(b)

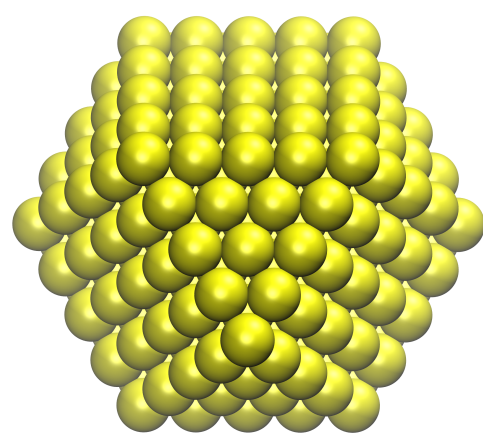

(d)

Figure S11: Comparison of distributions of $(\mathrm{a}, \mathrm{b})$ the methyl proton $P_{2}$ autocorrelation decay time scales $\left(\tau_{f}, \tau_{s}\right)$ and (c) computed $T_{2}$ relaxation time for different models of MTAB functionalized nanoparticle; MTAB65 is a spherical particle discussed in the main text, MTAB-facet is a 2-nm-diameter nanoparticle built using the CHARMM-GUI online server ${ }^{3}$ with Wulff construction (the core structure is shown in d); the latter features (100) and (111) surfaces and is functionalized with 61 MTAB ligands. 


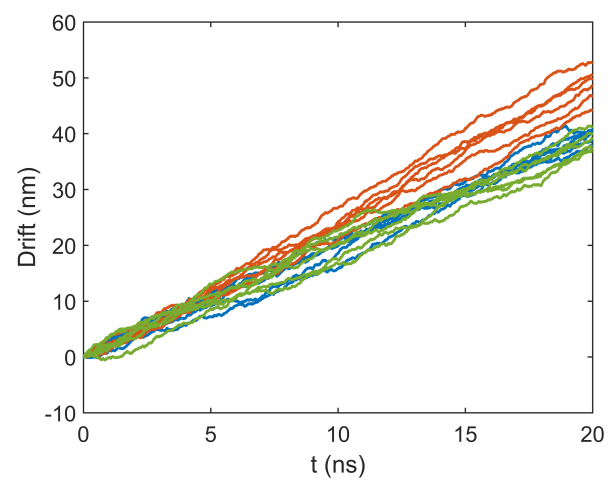

(a)

Figure S12: Examples of nanoparticle drift as a function of time from 30 ns drift velocity simulations. The different lines with the same color represent independent runs where the electric field is applied along $\pm \mathrm{x}, \pm \mathrm{y}, \pm \mathrm{z}$ directions. Color representation of different systems: red - MTAB65, blue - C4Q65, green - C4Q65 (15 nm box).

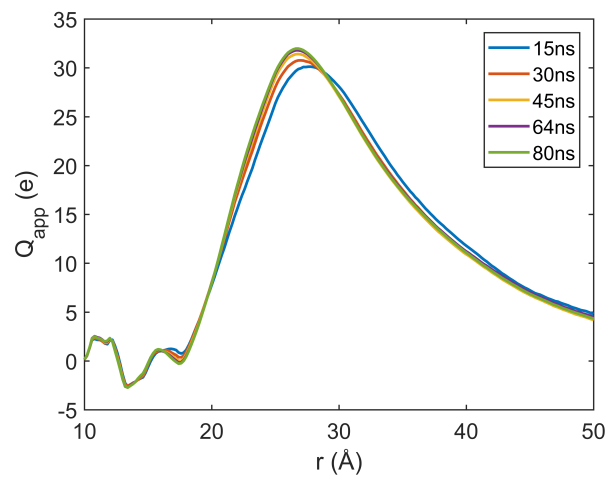

(a)

Figure S13: Convergence test of the effective charge profile $\left(Q_{a p p}(r)\right)$ for the MTAB65 system. The different curves show time-averaged profiles over different simulation lengths. 


\section{References}

(1) Wu, M.; Vartanian, A. M.; Chong, G.; Pandiakumar, A. K.; Hamers, R. J.; Hernandez, R.; Murphy, C. J. Solution NMR Analysis of Ligand Environment in Quaternary Ammonium-Terminated Self-Assembled Monolayers on Gold Nanoparticles: The Effect of Surface Curvature and Ligand Structure. J. Am. Chem. Soc. 2019, 141, 4316-4327.

(2) Virtanen, P.; Gommers, R.; Oliphant, T. E.; Haberland, M.; Reddy, T.; Cournapeau, D.; Burovski, E.; Peterson, P.; Weckesser, W.; J. Bright et al., SciPy 1.0: fundamental algorithms for scientific computing in Python. Nat. Methods 2020, 17, 261-272.

(3) Jo, S.; Kim, T.; Iyer, V. G.; Im, W. CHARMM-GUI: a web-based graphical user interface for CHARMM. J. Comput. Chem. 2008, 29, 1859-1865. 